\title{
Region of Dark Matter Present in the Hydrogen Atom
}

\section{Suto $\mathrm{K}^{*}$}

Independent Researcher, 5-24, Oote-Town, Isesaki, Japan

\begin{abstract}
This paper discusses ultra-low energy levels of the hydrogen atom which was not predictable with quantum mechanics. In the energy-momentum relationship applicable to the electron in a hydrogen atom, derived by the author, there is a negative energy solution, just like the Einstein relationship which holds in free space. The matter formed from a proton with positive mass, and an electron with negative mass that orbits near that proton, is smaller than an ordinary hydrogen atom to an extreme degree. When this unknown matter gathers in large amounts, it becomes a huge mass. This paper identifies such matter as the true nature of dark matter, the mysterious matter that physicists are currently searching for.
\end{abstract}

Keywords: Hydrogen atom; Ultra-low energy levels; Dark matter; Electron with negative mass; Triplet production; Einstein's energymomentum relationship; Dirac's relativistic wave equation

\section{Introduction}

In 1913, Bohr derived an equation for the energy levels of the hydrogen atom by assuming quantum condition, and thereby explained the stability of the atom. Later, Dirac derived the relativistic wave equation, and obtained more precise solutions for energy levels which incorporates spin. Incidentally, one of the most important relationships in the Special Theory of Relativity (STR) is as follows:

$$
\left(m_{0} c^{2}\right)^{2}+p^{2} c^{2}=\left(m_{0} c^{2}\right)^{2}
$$

Here, $m c^{2}$ is the relativistic energy of an object or a particle, and $m_{0} c^{2}$ is the rest mass energy. Currently, Einstein's relationship (1) is used to describe the energy and momentum of particles in free space, but for explaining the behavior of bound electrons inside atoms, opinion has shifted to quantum mechanics as represented by equations such as the Dirac's relativistic wave equation. For reasons such as these, there was no search for a relationship between energy and momentum applicable to an electron in the hydrogen atom. However, the author has ventured to take up this problem, and derived the following relationship [1].

$$
E_{\mathrm{re}, n}^{2}+\boldsymbol{p}_{n}^{2} c^{2}=\left(m_{\mathrm{e}} c^{2}\right)^{2}, n=1,2, \cdots .
$$

Here, $E_{r e, n}$ is the relativistic energy of the electron, described with an absolute scale. Also, $n$ is the total quantum number. From eqns. (1) and (2) it is evident that, if a stationary electron begins to move in free space, or is incorporated into an atom, then the energy which serves as the departure point is the rest mass energy. Consider the case where an electron currently stationary in free space is drawn to a proton to form a hydrogen atom. At this time, the rest mass energy of the electron decreases. We take this decrease in energy to be $-\Delta m_{e} c^{2}$, the energy of the photon emitted by the electron to be $h v$, and the kinetic energy gained by the electron to be $K$. For the law of conservation of energy to hold, the following relation must hold between these energies.

$$
-\Delta m_{\mathrm{e}} c^{2}+h v+K=0 .
$$

The author presented the following equation as an equation indicating the relationship between the rest mass energy and potential energy of the electron in a hydrogen atom [2].

$$
V(r)=-\Delta m_{\mathrm{e}} c^{2}
$$

According to this equation, the potential energy of a bound electron in a hydrogen atom is equal to the reduction in rest mass energy of that electron. When describing the motion of a bound electron in a hydrogen atom, a term must be included in that equation for the potential energy. From this $E_{\mathrm{re}, n}$ can be defined as follows.

$$
E_{\mathrm{re}, n}=m_{\mathrm{re}, n} c^{2}=m_{\mathrm{e}} c^{2}-h v=m_{\mathrm{e}} c^{2}+V\left(r_{n}\right)+K_{n}, n=1,2, \cdots .
$$

Here, $m_{\mathrm{re}, n}$ is the relativistic mass of the electron.

Incidentally, according to the virial theorem, the following relation holds between $K$ and $V$ :

$$
\langle K\rangle=-\frac{1}{2}\langle V\rangle \text {. }
$$

Here, $K$ is the kinetic energy of the entire system, and $V$ is the potential energy of the entire system. The average time of $K$ is equal to $-1 / 2$ the time average of $V$. Also, the sum of the time average $K$ and the time average of the total mechanical energy $E$ of the entire system becomes 0 . That is,

$$
\langle K\rangle+\langle E\rangle=0 \text {. }
$$

Next, if eqns. (6) and (7) are combined, the result is as follows:

$$
\langle E\rangle=-\langle K\rangle=\frac{1}{2}\langle V\rangle \text {. }
$$

Due to the above, $E_{\mathrm{re}, n}$ was defined classically as follows:

$$
E_{\mathrm{re}, n}=m_{\mathrm{e}} c^{2}+K_{n}+V\left(r_{n}\right)=m_{\mathrm{e}} c^{2}-K_{n}=m_{\mathrm{e}} c^{2}+E_{n} .
$$

The following section considers unknown energy levels that can be derived from eqn. (2).

\section{Unknown Energy Levels of the Hydrogen Atom that Cannot be Predicted with Quantum Mechanics}

Potential energy is not incorporated into eqn. (2) in a form which is visible to the eye. However, a quantity corresponding to $V\left(r_{n}\right)$ is incorporated from the beginning into $E_{r e, n}$ due to the definition of eqn. (5). There is a lower limit to potential energy, and the range which energy can assume is as follows.

*Corresponding author: Suto K, Independent Researcher, 5-24, Oote-Town Isesaki, 372-0048, Japan, Tel: 011+81-270-23-9980; E-mail: koshun_suto129@mbr.nifty.com

Received October 05, 2017; Accepted October 31, 2017; Published December 06, 2017

Citation: Suto K (2017) Region of Dark Matter Present in the Hydrogen Atom. J Phys Math 8: 252. doi: 10.4172/2090-0902.1000252

Copyright: () 2017 Suto K. This is an open-access article distributed under the terms of the Creative Commons Attribution License, which permits unrestricted use, distribution, and reproduction in any medium, provided the original author and source are credited. 


$$
-m_{\mathrm{e}} c^{2} \leq V(r)<0
$$

Also, the following constraint holds regarding the relativistic energy $E_{r e n}$ of the electron due to eqns. (8) and (10) (here, the discussion is limited to the ordinary energy levels of the atom).

$\frac{1}{2} m_{\mathrm{e}} c^{2} \leq E_{\mathrm{re}, n}<m_{\mathrm{e}} c^{2}$

Incidentally, it is known that the following formula can be derived from eqn. (1).

$$
E= \pm m_{0} c^{2}\left(1-\frac{v^{2}}{c^{2}}\right)^{-1 / 2}
$$

If the same logic is applied to eqn. (2), then the following formula can be derived.

$$
E_{\mathrm{re}}= \pm m_{\mathrm{e}} c^{2}\left(1+\frac{v^{2}}{c^{2}}\right)^{-1 / 2}
$$

Next, when a Taylor expansion is performed on eqn. (13),

$$
E_{\text {re }} \approx \pm m_{\mathrm{e}} c^{2}\left(1-\frac{v^{2}}{2 c^{2}}+\frac{3 v^{4}}{8 c^{4}}\right)
$$

According to quantum mechanics textbooks, the eigenvalue of the energy of a hydrogen atom as obtained from the Dirac's relativistic wave equation is as follows [3].

$$
E=m_{\mathrm{e}} c^{2}\left[1-\frac{\gamma^{2}}{2 n^{2}}-\frac{\gamma^{4}}{2 n^{4}}\left(\frac{n}{|k|}-\frac{3}{4}\right)\right] .
$$

It is important to note that energy here is defined on an absolute scale. Because $\mathrm{Z}=1$ in the case of a hydrogen atom, $\gamma=e^{2} / \hbar c$ ( $\gamma$ is the fine structure constant $\alpha$ ).

The factor $\alpha$ is a dimensionless constant as follows.

$$
\alpha=\frac{e^{2}}{4 \pi \varepsilon_{0} \hbar c}=7.2973525664 \times 10^{-3} \text {. }
$$

Thus this paper makes the following assumption, based on a comparison of eqns. (14) and (15).

$$
\frac{v_{n}}{c}=\frac{\alpha}{n}, \quad n=1,2, \cdots .
$$

However, the velocity is taken to be the average velocity of the electron, in accordance quantum mechanics. Here, $v$ on the left side was set to $v_{n}$.

When this is done, the following $E_{\mathrm{re}, n}$ and $E_{n}$ can be derived from eqns. (13) and (9).

$$
\begin{aligned}
& E_{\mathrm{re}, n}= \pm m_{\mathrm{re}, n} c^{2}= \pm m_{\mathrm{e}} c^{2}\left(\frac{n^{2}}{n^{2}+\alpha^{2}}\right)^{1 / 2}, \quad n=1,2, \cdots . \\
& E_{n}=m_{\mathrm{e}} c^{2}\left[\left(\frac{n^{2}}{n^{2}+\alpha^{2}}\right)^{1 / 2}-1\right] .
\end{aligned}
$$

$E$ of eqn. (15) and $E_{\mathrm{re}, n}$ of eqn. (18) define an absolute quantity, which includes the electron's rest mass energy. Where as $E_{n}$ in eqn. (19) expresses the reduction in rest mass energy of the electron.

Eqn. (17) is a replacement necessary for shifting from classical mechanics, which describes values of continuous physical quantities, to quantum theory, which treats values of discrete physical quantities.

Incidentally, the equation derived from classical quantum theory is following:

$$
E_{\mathrm{B}, n}=-\frac{1}{2}\left(\frac{1}{4 \pi \varepsilon_{0}}\right)^{2} \frac{m_{\mathrm{e}} e^{4}}{\hbar^{2}} \cdot \frac{1}{n^{2}}=-\frac{\alpha^{2}}{2 n^{2}} m_{\mathrm{e}} c^{2}, \quad n=1,2, \cdots .
$$

Here, the $\mathrm{B}$ in $E_{\mathrm{B}, n}$ indicates the equation derived by Bohr.

Now, if a Taylor expansion is performed on the right side of eqn. (19),

$E_{n}=m_{\mathrm{e}} c^{2}\left[\left(1+\frac{\alpha^{2}}{n^{2}}\right)^{-1 / 2}-1\right] \approx m_{\mathrm{e}} c^{2}\left[\left(1-\frac{\alpha^{2}}{2 n^{2}}+\frac{3 \alpha^{4}}{8 n^{4}}\right)-1\right] \approx m_{\mathrm{e}} c^{2}\left(-\frac{\alpha^{2}}{2 n^{2}}+\frac{3 \alpha^{4}}{8 n^{4}}\right)$.

When this is done, the equations for the energies is as follows.

$E_{n} \approx-\frac{\alpha^{2}}{2 n^{2}} m_{\mathrm{e}} c^{2}$.

From this, it is evident that Bohr's energy equation, eqn. (20), is an approximation of eqn. (19).

The following compares energies when $n=1$.

Value predicated by this paper eqn. (19): $\quad=-13.60515 \mathrm{eV}$. (23a)

Value predicted by Bohr eqn. (20): $E_{\mathrm{B}, 1}=-13.60569 \mathrm{eV}$.

$\frac{E_{\mathrm{B}, 1}}{E_{1}}=1.0000397$.

Incidentally, there are also positive and negative solutions for $E_{\mathrm{re}, n}$ in eqn. (2). Here, the ordinary, known energies of a hydrogen atom are expressed as $E_{\mathrm{re}, n}^{+}, E_{n}^{+}$. Also, the negative energies are expressed as $E_{\mathrm{re}, n}^{-}, E_{n}^{-}$.

The equations for positive energies is as follows.

$$
E_{\mathrm{re}, n}^{+}=m_{\mathrm{e}} c^{2}\left(1+\frac{\alpha^{2}}{n^{2}}\right)^{-1 / 2} \approx m_{\mathrm{e}} c^{2}-\frac{\alpha^{2}}{2 n^{2}} m_{\mathrm{e}} c^{2}, \quad n=1,2, \cdots
$$

In contrast, the equations for the negative solutions are as follows.

$$
\begin{aligned}
& E_{\mathrm{re}, n}^{-}=-m_{\mathrm{e}} c^{2}\left(1+\frac{\alpha^{2}}{n^{2}}\right)^{-1 / 2} \approx-m_{\mathrm{e}} c^{2}+\frac{\alpha^{2}}{2 n^{2}} m_{\mathrm{e}} c^{2}, \quad n=1,2, \cdots \\
& E_{n}^{-} \approx-2 m_{\mathrm{e}} c^{2}+\frac{\alpha^{2}}{2 n^{2}} m_{\mathrm{e}} c^{2} .
\end{aligned}
$$

If the relativistic mass of the electron is taken to be $m_{\mathrm{re}, n}$, then the negative solution can be written as follows:

$$
m_{\mathrm{re}, n}^{-}=-m_{\mathrm{e}}\left(1-\frac{\alpha^{2}}{2 n^{2}}\right)<0 .
$$

From this it can be predicted that an electron at the energy levels in eqn. (25) has negative mass.

\section{Classical Orbital Radii of the Electron at the Unknown Energy Levels}

This section discusses the unknown orbital radii of a hydrogen atom. Incidentally, the energy of the hydrogen atom can also be written as follows.

$$
E_{n}=-\frac{1}{2} \frac{1}{4 \pi \varepsilon_{0}} \frac{e^{2}}{r_{n}}=-\frac{1}{2} \frac{r_{\mathrm{e}} m_{\mathrm{e}} c^{2}}{r_{n}}=-m_{\mathrm{e}} c^{2}\left(\frac{r_{\mathrm{e}} / 2}{r_{n}}\right) .
$$

Here, $r$ is the classical electron radius as follows.

$r_{\mathrm{e}}=\frac{e^{2}}{4 \pi \varepsilon_{0} m_{\mathrm{e}} c^{2}}=2.8179403227 \times 10^{-15} \mathrm{~m}$.

Also, the following equation for energy can be obtained from eqns. (9) and (28). 


$$
E_{\mathrm{re}, n}=m_{\mathrm{e}} c^{2}+E_{n}=m_{\mathrm{e}} c^{2}\left(1-\frac{r_{\mathrm{e}} / 2}{r_{n}}\right) .
$$
$E_{r e}=0$ is:

Here, if $-m_{\mathrm{e}} c^{2}$ is substituted for $E$ in eqn. (28), then the $r$ where

$$
r=\frac{r_{\mathrm{e}}}{2} .
$$

Here, $\left(r_{n}-r_{\mathrm{e}} / 2\right)$ is distance in the $0 \leq E_{\mathrm{re}}$ region within the orbital radii $r_{n}$ (In contrast, $r_{\mathrm{e}} / 2$ is distance in the $E_{\mathrm{re}} \leq 0$ region). In quantum mechanics, $r$ is an average value not a definitive value, and this paper follows that principle.

Dirac pointed out that there is a negative solution to eqn. (1). Adopting the same viewpoint, there is a negative solution to eqn. (2). To find the negative solution, it is necessary to create a quadratic equation for $r$. Thus, from eqns. (18) and (30),

$$
\left(\frac{r_{n}-r_{\mathrm{e}} / 2}{r_{n}}\right)^{2}=\frac{n^{2}}{n^{2}+\alpha^{2}} \text {. }
$$

From this, the following quadratic equation is obtained.

$$
r_{n}^{2}-\left(\frac{n^{2}+\alpha^{2}}{\alpha^{2}}\right) r_{\mathrm{e}} r_{n}+\left(\frac{n^{2}+\alpha^{2}}{\alpha^{2}}\right) \frac{r_{\mathrm{e}}^{2}}{4}=0 .
$$

If this equation is solved for $r_{n}$

$$
r_{n}=\frac{r_{\mathrm{e}}}{2}\left(1+\frac{n^{2}}{\alpha^{2}}\right)\left[1 \pm\left(1+\frac{\alpha^{2}}{n^{2}}\right)^{-1 / 2}\right]
$$

When the Taylor expansion of eqn. (34) is taken, the result is as follows.

$$
r_{n} \approx \frac{r_{\mathrm{e}}}{2}\left(1+\frac{n^{2}}{\alpha^{2}}\right)\left[1 \pm\left(1-\frac{\alpha^{2}}{2 n^{2}}+\frac{3 \alpha^{4}}{8 n^{4}}\right)\right]
$$

To begin, the positive solution is found first. (The positive solution is the solution found by Bohr.) The radii $r_{n}^{+}$found from eqn. (35) are as follows.

$$
r_{n}^{+} \approx \frac{3 r_{\mathrm{e}}}{4}+\frac{r_{\mathrm{e}}}{\alpha^{2}} n^{2}=\frac{3 r_{\mathrm{e}}}{4}+a_{\mathrm{B}} n^{2} .
$$

Here, $a_{\mathrm{B}}$ is the Bohr radius as follows.

$$
a_{\mathrm{B}}=\frac{4 \pi \varepsilon_{0} \hbar^{2}}{m_{\mathrm{e}} e^{2}}=0.52917721067 \times 10^{-10} \mathrm{~m} \text {. }
$$

In contrast, the radii $r_{n}$ found by Bohr are given by the following equation.

$$
r_{n}=a_{\mathrm{B}} n^{2}, n=1,2 \ldots
$$

If eqns. (36) and (38) are compared, it is evident that eqn. (38) is an approximation. Next, the negative solution $r_{n}^{-}$of eqn. (35),

$$
r_{n}^{-} \approx \frac{r_{\mathrm{e}}}{4}+\frac{\alpha^{2} r_{\mathrm{e}}}{16 n^{2}}=\frac{r_{\mathrm{e}}}{4}+\frac{a_{\mathrm{B}}}{n^{2}}\left(\frac{\alpha}{2}\right)^{4} .
$$

Since $r^{-}$converges to $r_{\mathrm{e}} / 4, r_{\mathrm{e}} / 4$ can be regarded as the radius of the atomic nucleus of a hydrogen atom (i.e., the proton). Here, the theoretical value of the proton radius is:

$$
\frac{r_{\mathrm{e}}}{4}=0.704485080675 \times 10^{-15} \mathrm{~m} \text {. }
$$

Incidentally, the orbital radii $r_{n}$ of the electron is a classical concept. In quantum mechanics, $r_{n}$ is defined in each stationary state as the radii where the probability that the electron is present is maximal. In this paper too, $r_{n}$ is used in the sense of quantum mechanics.

The next compares the orbital radii of an electron in a hydrogen atom $r_{n}^{+}$and the orbital radii of an electron with a negative mass $r_{n}^{-}$. Referring to eqn. (34),

$$
\frac{r_{n}^{-}}{r_{n}^{+}}=\left[1-\left(\frac{n^{2}}{n^{2}+\alpha^{2}}\right)^{1 / 2}\right]\left[1+\left(\frac{n^{2}}{n^{2}+\alpha^{2}}\right)^{1 / 2}\right]^{-1}=\frac{\left(n^{2}+\alpha^{2}\right)^{1 / 2}-n}{\left(n^{2}+\alpha^{2}\right)^{1 / 2}+n} .
$$

Here, if we set $n=1$,

$$
\frac{r_{1}^{-}}{r_{1}^{+}}=\frac{\left(1+\alpha^{2}\right)^{1 / 2}-1}{\left(1+\alpha^{2}\right)^{1 / 2}+1}=1.3312484168 \times 10^{-5} \text {. }
$$

Next, if $\left(r_{1}^{-}-r_{\mathrm{e}} / 4\right)$ and $r_{e} / 4$ are compared using $r_{1}^{-}$in eqn. (39).

$$
\frac{r_{1}^{-}-r_{\mathrm{e}} / 4}{r_{\mathrm{e}} / 4} \approx \frac{\alpha^{2}}{4}=1.33128 \times 10^{-5} \text {. }
$$

From this, it is evident that the electron with negative mass is located near the atomic nucleus. Based on eqns. (42) and (43), there is a possibility that the following relationship holds.

$\frac{r_{n}^{-}}{r_{n}^{+}}=\frac{r_{n}^{-}-r_{\mathrm{e}} / 4}{r_{\mathrm{e}} / 4}$

Let's check this eqn. (44) can be written as follows.

$\frac{r_{n}^{+}+r_{n}^{-}}{r_{n}^{+}}=\frac{r_{n}^{-}}{r_{\mathrm{e}} / 4}$.

From this,

$\frac{r_{\mathrm{e}}}{4}\left(r_{n}^{+}+r_{n}^{-}\right)=r_{n}^{+} r_{n}^{-}$.

First, from eqn. (34), the left side of eqn. (46) is,

$\frac{r_{\mathrm{e}}}{4}\left(r_{1}^{+}+r_{n}^{-}\right)=\left(\frac{r_{\mathrm{e}}}{2}\right)^{2}\left(1+\frac{n^{2}}{\alpha^{2}}\right)$.

Next, if eqn. (34) is used in the same way as the right side of eqn. (46),

$$
r_{n}^{+} r_{n}^{-}=\left(\frac{r_{\mathrm{e}}}{2}\right)^{2}\left(1+\frac{n^{2}}{\alpha^{2}}\right)^{2}\left[1-\left(\frac{n^{2}}{n^{2}+\alpha^{2}}\right)\right]=\left(\frac{r_{\mathrm{e}}}{2}\right)^{2}\left(1+\frac{n^{2}}{\alpha^{2}}\right) \text {. }
$$

From eqns. (47) and (48), it is evident that eqn. (44) holds. Also, the following equation can be derived from eqn. (44).

$$
\begin{aligned}
& r_{n}^{+}=\frac{r_{\mathrm{e}}}{4} \frac{r_{n}^{-}}{r_{n}^{-}-r_{\mathrm{e}} / 4} . \\
& r_{n}^{-}=\frac{r_{\mathrm{e}}}{4} \frac{r_{n}^{+}}{r_{n}^{+}-r_{\mathrm{e}} / 4} .
\end{aligned}
$$

Also, the following relationship is obtained from eqns. (41) and (44).

$$
\frac{r_{n}^{-}}{r_{n}^{+}}=\frac{\left(n^{2}+\alpha^{2}\right)^{1 / 2}-n}{\left(n^{2}+\alpha^{2}\right)^{1 / 2}+n}=\frac{r_{n}^{-}-r_{\mathrm{e}} / 4}{r_{\mathrm{e}} / 4} \text {. }
$$

When $r_{n}^{-}$is found from eqn. (51), the following two equations are obtained.

$$
r_{n}^{-}=r_{n}^{+} \cdot \frac{\left(n^{2}+\alpha^{2}\right)^{1 / 2}-n}{\left(n^{2}+\alpha^{2}\right)^{1 / 2}+n}
$$




$$
r_{n}^{-}=\frac{r_{\mathrm{e}}}{2} \frac{\left(n^{2}+\alpha^{2}\right)^{1 / 2}}{\left(n^{2}+\alpha^{2}\right)^{1 / 2}+n} \text {. }
$$

The following relationship holds due to eqns. (50) and (52).

$$
\frac{r_{\mathrm{e}}}{4} \frac{1}{r_{n}^{+}-r_{\mathrm{e}} / 4}=\frac{\left(n^{2}+\alpha^{2}\right)^{1 / 2}-n}{\left(n^{2}+\alpha^{2}\right)^{1 / 2}+n} \text {. }
$$

When $r_{n}^{+}$is found from eqn. (54),

$$
r_{n}^{+}=\frac{r_{\mathrm{e}}}{2} \frac{\left(n^{2}+\alpha^{2}\right)^{1 / 2}}{\left(n^{2}+\alpha^{2}\right)^{1 / 2}-n} \text {. }
$$

Here, if we set $n=1$ eqn. (55) is,

$$
r_{1}^{+}=0.52919834512 \times 10^{-10} \mathrm{~m} \text {. }
$$

Next, if $r_{1}^{+}$and Bohr radius are compared,

$$
\frac{r_{1}^{+}}{a_{\mathrm{B}}}=1.0000399383 \text {. }
$$

Rearranging eqns. (55) and (53), we obtain the following equation.

$$
\begin{aligned}
& r_{n}^{+}=\frac{r_{\mathrm{e}}}{2}\left[1-\left(\frac{n^{2}}{n^{2}+\alpha^{2}}\right)^{1 / 2}\right]^{-1}, n=1,2, \cdots . \\
& r_{n}^{-}=\frac{r_{\mathrm{e}}}{2}\left[1+\left(\frac{n^{2}}{n^{2}+\alpha^{2}}\right)^{1 / 2}\right]^{-1}, n=1,2, \cdots .
\end{aligned}
$$

Here, when $n=1$ eqn. (59) is,

$$
r_{1}^{-}=7.0449445912 \times 10^{-16} \mathrm{~m} \text {. }
$$

Next, if $r_{1}^{-}$and the proton radius are compared,

$$
\frac{r_{1}^{-}}{r_{\mathrm{e}} / 4}=1.0000133124
$$

Also, if eqns. (49) and (50) are combined,

$$
\frac{r_{n}^{-}}{r_{n}^{+}}=\frac{r_{\mathrm{e}} / 4}{r_{n}^{+}-r_{\mathrm{e}} / 4}=\frac{r_{n}^{-}-r_{\mathrm{e}} / 4}{r_{\mathrm{e}} / 4} \text {. }
$$

Here, when $n=1$ eqn. (62) is,

$$
\frac{r_{1}^{-}}{r_{1}^{+}}=\frac{r_{\mathrm{e}} / 4}{r_{1}^{+}-r_{\mathrm{e}} / 4}=\frac{r_{1}^{-}-r_{\mathrm{e}} / 4}{r_{\mathrm{e}} / 4}=\frac{\left(1+\alpha^{2}\right)^{1 / 2}-1}{\left(1+\alpha^{2}\right)^{1 / 2}+1}=1.3312484168 \times 10^{-5} \text {. }
$$

From eqns. (53) and (55), the following relationships hold between $r_{n}^{+}$and $r_{n}^{-}$.

$$
\begin{aligned}
& r_{n}^{+}+r_{n}^{-}=r_{\mathrm{e}}\left(1+\frac{n^{2}}{\alpha^{2}}\right) . \\
& r_{n}^{+}-r_{n}^{-}=\frac{r_{\mathrm{e}}}{\alpha^{2}} n^{2}\left(1+\frac{\alpha^{2}}{n^{2}}\right)^{1 / 2}=a_{\mathrm{B}} n^{2}\left(1+\frac{\alpha^{2}}{n^{2}}\right)^{1 / 2} \approx a_{\mathrm{B}} n^{2} .
\end{aligned}
$$

Incidentally, the author has previously derived the following equation [4].

$$
\frac{E_{\mathrm{re}, n}}{m_{\mathrm{e}} c^{2}}=\frac{n}{\left(n^{2}+\alpha^{2}\right)^{1 / 2}}=\frac{m_{\mathrm{re}, n}}{m_{\mathrm{e}}}=\frac{r_{n}-r_{\mathrm{e}} / 2}{r_{n}} .
$$

If eqns. (65) and (66) are combined,

$$
\frac{n}{\left(n^{2}+\alpha^{2}\right)^{1 / 2}}=\frac{m_{\mathrm{re}, n}}{m_{\mathrm{e}}}=\frac{r_{n}^{+}-r_{\mathrm{e}} / 2}{r_{n}^{+}}=\frac{a_{\mathrm{B}} n^{2}}{r_{n}^{+}-r_{n}^{-}} \text {. }
$$

The following relationship is also obtained from eqn. (62).

$$
\left(r_{n}^{+}-\frac{r_{\mathrm{e}}}{4}\right)\left(r_{n}^{-}-\frac{r_{\mathrm{e}}}{4}\right)=\left(\frac{r_{\mathrm{e}}}{4}\right)^{2} \text {. }
$$

Incidentally, there are vast clouds of hydrogen gas around the galaxy clusters that exist in space. The energy of matter made up of an electron with negative mass and a proton with positive mass is far less than the energy of an ordinary hydrogen atom. Naturally, this unknown matter exists in larger amounts than hydrogen gas. In addition, it is far smaller in size an ordinary hydrogen atom, and thus if these atoms gather in large amounts, they become a tremendous source of gravity. This matter is electrically neutral and stable. It is also extremely small in size, and therefore can pass through ordinary matter. Thus, in this paper the system of an electron with negative mass and a proton with positive mass is regarded as the true nature of dark matter, the mysterious matter whose nature is unknown.

\section{Discussion}

A) The radius $r$ where $E_{\mathrm{re}}=0$ is $r_{\mathrm{e}} / 2$ due to eqn. (30). Dirac predicted that the vacuum energy $E_{\mathrm{re}}^{\mathrm{re}}$ satisfies the relation $E_{\mathrm{re}}<-m_{\mathrm{e}} c^{2}$, but actually $E_{\mathrm{re}}=0$ is the energy of the virtual electron-positron pair which make up the vacuum (Figure 1).

The relationship between $r$ and $E_{\mathrm{re}}$ is summarized as follows.

Region A: $r_{\mathrm{e}}<r_{n}^{+}<\infty \leftrightarrow \frac{1}{2} m_{\mathrm{e}} c^{2}<E_{\mathrm{re}}^{+}<m_{\mathrm{e}} c^{2}$.

Classically, this is the region where electrons can exist inside a hydrogen atom.

Region B: $\frac{r_{\mathrm{e}}}{2} \leq r_{\mathrm{vp}} \leq r_{\mathrm{e}} \leftrightarrow 0 \leq E_{\mathrm{vp}} \leq m_{\mathrm{e}} c^{2}, \quad\left(0 \leq E_{\mathrm{re}} \leq \frac{m_{\mathrm{e}} c^{2}}{2}\right)$.

Eqns. (18) and (58) have a solution with $n=0$ (thus far, this paper

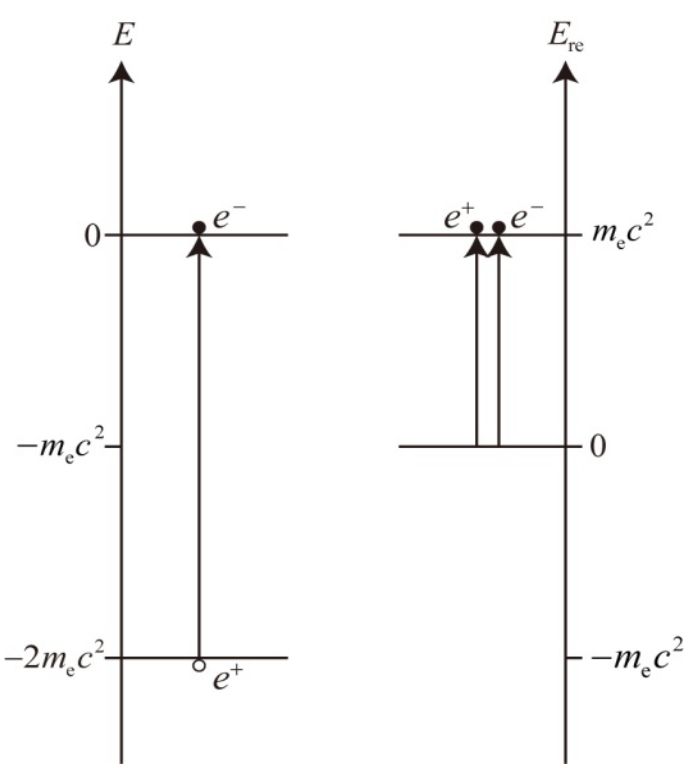

Figure 1: Differences between Dirac's hole theory and the interpration in this paper. 
has treated the problem of the hydrogen atom, so the case where $n=0$ was omitted). That is, in eqn. (18) $E_{\mathrm{re}, 0}=0$ and in eqn. (58) $r_{0}=r_{\mathrm{e}} / 2$.

However, as is evident from eqn. (11), it is impossible classically for an electron to be present in region B (of course, in quantum mechanics, the electron is described by a wave function, and hence the probability that the electron is present at $r=r_{\mathrm{e}} / 2$ is not zero.)

Thus, this paper predicts that classically this is the region where virtual particle pairs exist (i.e., pairs of a virtual electron and virtual positron with zero mass) [5]. If energy of $2 m_{\mathrm{e}} c^{2}$ or more is applied to the virtual particle pairs $\left(E_{\mathrm{vp}}=0\right)$ at $r=r_{\mathrm{e}} / 2$ then an electron and positron are produced. Here, $E_{\mathrm{vp}}$ is the energy of the virtual particle pair ("vp" stands for "virtual particle"). Here, the energy $E_{\mathrm{vp}}$ of the virtual particle pair is assumed to be 2 times that of the virtual electron energy $E_{\mathrm{re}}$. Incidentally, according to the qualitative interpretation of the uncertainty principle, it is forbidden for the electron to approach the atomic nucleus, and for the momentum of the electron to approach zero. If a particle is confined in a small area, the momentum of the particle increases. However, in region $\mathrm{B}$, the uncertainty principle does not hold in this sense. When the location where the virtual particle pair exists approaches from $r_{\mathrm{e}}$ to $r_{\mathrm{e}} / 2$, the momentum of the virtual particle pair approaches zero. In other words,

$$
r_{\mathrm{e}} \rightarrow r_{\mathrm{e}} / 2, p \rightarrow 0 \text {. }
$$$$
\text { Region C: } \frac{r_{\mathrm{e}}}{3} \leq r_{\mathrm{vp}}<\frac{r_{\mathrm{e}}}{2} \leftrightarrow-m_{\mathrm{e}} c^{2} \leq E_{\mathrm{vp}}<0
$$

This is the region of the virtual particle pair with negative mass.

$$
\text { Region D: } \frac{r_{\mathrm{e}}}{4}<r_{n}^{-}<\frac{r_{\mathrm{e}}}{3} \leftrightarrow-m_{\mathrm{e}} c^{2}<E_{\mathrm{re}, n}^{-}<-\frac{1}{2} m_{\mathrm{e}} c^{2} .
$$

In this region, there is an electron with negative mass. Unknown matter formed from a proton with positive mass and an electron with negative mass is lighter in mass than an ordinary hydrogen atom by about the mass of two electrons. However, this matter is far smaller than the hydrogen atom, and when it gathers in large amounts, it can become a tremendous source of gravity. The author predicts that one of the two electrons produced in triplet production experiments is an electron which had negative mass [6-7]. The regions where eqn. (2) is applicable are $\mathrm{A}$ and $\mathrm{D}$.

$$
\text { Region E: } r<\frac{r_{\mathrm{e}}}{4} \leftrightarrow E_{\mathrm{re}}<-m_{\mathrm{e}} c^{2}
$$

According to quantum mechanics, the region where $E_{\mathrm{re}}<-m_{\mathrm{e}} c^{2}$ is the region of the particle with negative energy in eqn. (1) (region of the Dirac sea). However, according to this paper, the region $\mathrm{E}$ is a region in the atomic nucleus.

B) The proton is not direct concern of this paper, but it is important, so here it will be discussed. The fact that, in this paper, we were able to theoretically predict the radius of the proton is decisively important. Normally, a quantum mechanical particle is regarded as behaving like a wave until it is observed, and like a particle after it is observed. However, according to this paper, a proton exists as a localized particle even if it is not observed.

The double-slit experiment is an experiment which shows the strangeness of quantum mechanics. Ordinarily, this experiment is carried out using photons or electrons. If this thought experiment is carried out using protons, what will happen? In this case, the protons of radius $r / 4$ should pass through either of the two slits. Nevertheless, the protons with wave characteristics will interfere with themselves. We can predict that interference fringes will appear on the screen behind.
However, in the traditional interpretation of quantum mechanics, this is explained as a single particle behaving as if it had passed simultaneously through two slits. In quantum mechanics, they do not say that the particle had to pass through one slit or other. However, if it assumed that a proton exists with a certain size at a certain location in space, then the traditional interpretation of quantum mechanics (Copenhagen interpretation) must be corrected.

\section{Conclusion}

The hydrogen atom have energy levels far lower than the energy levels that can be derived from quantum mechanics. That is,

$$
E_{\mathrm{re}, n}^{-}=-m_{\mathrm{e}} c^{2}\left(\frac{n^{2}}{n^{2}+\alpha^{2}}\right)^{1 / 2} \approx-m_{\mathrm{e}} c^{2}+\frac{\alpha^{2}}{2 n^{2}} m_{\mathrm{e}} c^{2}, \quad n=1,2, \cdots .
$$

The classical orbital radii of the electron at the energy levels in eqn. (74) is given by the following equation.

$$
\begin{aligned}
& r_{n}^{-}=\frac{r_{\mathrm{e}}}{4} \frac{r_{n}^{+}}{r_{n}^{+}-r_{\mathrm{e}} / 4} . \\
& r_{n}^{-}=\frac{r_{\mathrm{e}}}{2}\left[1+\left(\frac{n^{2}}{n^{2}+\alpha^{2}}\right)^{1 / 2}\right]^{-1}, n=1,2, \cdots .
\end{aligned}
$$

When we set $n=1$ in eqn. (76),

$$
r_{1}^{-}=7.0449445912 \times 10^{-16} \mathrm{~m} \text {. }
$$

Also,

$$
\frac{r_{1}^{-}}{r_{\mathrm{e}} / 4}=1.0000133124
$$

An electron at the energy levels in eqn. (74) exists near the atomic nucleus. The question of what to call this system of a proton and electron will be an issue for the future.

$$
\begin{aligned}
& r_{n}^{-} \text {is far smaller than } r_{n}^{+} . \text {That is, } \\
& \frac{r_{1}^{-}}{r_{1}^{+}}=1.3312484168 \times 10^{-5} .
\end{aligned}
$$

The following relationships hold between $r_{n}^{+}$and $r_{n}^{-}$.

$$
\begin{aligned}
& r_{n}^{+}+r_{n}^{-}=r_{\mathrm{e}}\left(1+\frac{n^{2}}{\alpha^{2}}\right), n=0,1,2, \cdots \\
& r_{n}^{+}-r_{n}^{-}=\frac{r_{\mathrm{e}}}{\alpha^{2}} n^{2}\left(1+\frac{\alpha^{2}}{n^{2}}\right)^{1 / 2}=a_{\mathrm{B}} n^{2}\left(1+\frac{\alpha^{2}}{n^{2}}\right)^{1 / 2} \approx a_{\mathrm{B}} n^{2}, n=0,1,2, \cdots \\
& r_{n}^{+} r_{n}^{-}=\left(\frac{r_{\mathrm{e}}}{2}\right)^{2}\left(1+\frac{n^{2}}{\alpha^{2}}\right), n=0,1,2, \cdots \\
& \left(r_{n}^{+}-\frac{r_{\mathrm{e}}}{4}\right)\left(r_{n}^{-}-\frac{r_{\mathrm{e}}}{4}\right)=\left(\frac{r_{\mathrm{e}}}{4}\right)^{2}, n=0,1,2, \cdots \\
& \frac{r_{n}^{-}}{r_{n}^{+}}=\frac{r_{\mathrm{e}} / 4}{r_{n}^{+}-r_{\mathrm{e}} / 4}=\frac{r_{n}^{-}-r_{\mathrm{e}} / 4}{r_{\mathrm{e}} / 4}=\frac{\left(n^{2}+\alpha^{2}\right)^{1 / 2}-n}{\left(n^{2}+\alpha^{2}\right)^{1 / 2}+n}, n=0,1,2, \cdots . \\
& \frac{n}{\left(n^{2}+\alpha^{2}\right)^{1 / 2}}=\frac{m_{\mathrm{re}, n}}{m_{\mathrm{e}}}=\frac{r_{n}^{+}-r_{\mathrm{e}} / 2}{r_{n}^{+}}=\frac{a_{\mathrm{B}} n^{2}}{r_{n}^{+}-r_{n}^{-}}, n=1,2, \cdots .
\end{aligned}
$$

The above relationships can also be applied to a virtual particle pair, and thus $n=0$ is included. The energy of matter made up of an electron with negative mass and a proton with positive mass is far less than the 
energy of an ordinary hydrogen atom. In addition, it is far smaller in size an ordinary hydrogen atom, and thus if these atoms gather in large amounts, they become a tremendous source of gravity.

Thus, in this paper the system of an electron with negative mass and a proton with positive mass is regarded as the true nature of dark matter, the mysterious matter whose nature is unknown.

\section{References}

1. Suto K (2011) An energy-momentum relationship for a bound electron inside a hydrogen atom. Physics Essays 24: 301-307.

2. Suto K (2009) True nature of potential energy of a hydrogen atom. Physics Essays 22: 135-139.
3. Schiff LI (1968) Quantum Mechanics. McGraw-Hill, New York.

4. Suto K (2017) Elucidating the Nature of the Fine Structure Constant and Indicating the Existence of an Unknown Angular Momentum. Applied Physics Research 9: 7-16.

5. Suto K (2017) Previously unknown physical formulas which hold in a hydrogen atom and are derived without using quantum mechanics. Applied Physics Research. 9: 17-22.

6. Suto K (2017) Presentation of dark matter candidates. Applied Physics Research 9: 70-76.

7. Suto K (2014) Previously unknown ultra-low energy level of the hydrogen atom whose existence can be predicted. Applied Physics Research 6: 64-73. 